\title{
Las Normas Internacionales de Información Financiera y su relevancia informativa: evidencia empírica en empresas cotizadas de Colombia
}

\author{
The International Financial Reporting Standards and their informative relevance: \\ Empirical evidence of Colombia listed companies \\ Diego Fernando Católico Segura* \\ Universidad de La Salle, Colombia
}

Recibido el 11 de enero de 2019; aceptado el 4 de mayo de 2020

Disponible en Internet el: 10 de junio de 2020

\section{Resumen}

Este artículo expone el efecto de la adopción de las Normas Internacionales de Información Financiera (NIIF) en la revelación de información en empresas cotizadas en Colombia y examina la asociación entre el nivel de cumplimiento de los requisitos de revelación de las NIIF y las características de las empresas, como posibles impulsores adicionales de la revelación de información. Se realiza un análisis de contenido de las notas de los estados financieros publicados en los años 2014 y 2015 de compañías cotizadas colombianas para observar el cumplimiento de los requisitos de revelación y se hace uso de un modelo de Mínimos Cuadrados Ordinarios con datos de corte transversal para establecer sus posibles determinantes. El estudio evidenció una mejoría en la revelación de la información contable, a partir de la adopción de las NIIF y se determinó que la internacionalización de las compañías tiene una relación positiva y significativa con la mayor revelación de información.

Código JEL: F20, M41, M48

Palabras clave: Normas Internacionales de Información Financiera; NIIF; Revelación de información; Calidad de la información contable; Determinantes

\footnotetext{
${ }^{*}$ Autor para correspondencia

Correo electrónico: dfcatolico@unisalle.edu.co (D. F. Católico Segura).

La revisión por pares es responsabilidad de la Universidad Nacional Autónoma de México. 


\begin{abstract}
This article exposes the effect of the adoption of the International Financial Reporting Standards (IFRS) in the disclosure of information of Colombia listed companies and examine the association between the level of compliance of IFRS and firm characteristics, as possible additional drivers of disclosure. The study uses a content analysis of the notes of the financial statements published in 2014 and 2015 of Colombian companies to observe compliance with the disclosure requirements and used a model of Ordinary Minimum Squares with cross-sectional data to establish its possible determinants. The study showed an improvement in the disclosure of accounting information from the adoption of IFRS and it was determined that the internationalization of companies has a positive and significant relationship with the level disclosure of information.
\end{abstract}

JEL Code: F20, M41, M48

Keywords: International Financial Reporting Standards; IFRS; Disclosure of Information; Accounting information quality; Determinants

\title{
Introducción
}

La adopción de las Normas Internacionales de Información Financiera ha traído consigo cambios significativos al momento de generar y presentar la información contable. Tal situación es consecuencia de la aplicación de nuevos criterios de reconocimiento, medición, presentación y revelación de la información. Estos cambios requieren del estudio sobre sus consecuencias e impactos reales para evaluar la adecuada configuración y aplicación de dichos lineamientos y de los beneficios que han traído en el ámbito informativo para las empresas y sus grupos de interés.

Pascan (2015) considera que, con el fin de lograr los beneficios esperados de la adopción de las NIIF, relacionados con una mayor comparabilidad y transparencia de los informes financieros, la aplicación de estas normas debería dar como resultado una mejor calidad de la información contable. Autores como Barth, Landsman y Lang (2008) y Iatridis (2010) han evidenciado empíricamente que el seguir las NIIF llevan a la obtención de información de mayor calidad. Por su parte, Cutillas, Sánchez y Yague (2016) han señalado que una vez adoptadas las NIIF se evidencia un incremento en la relevancia valorativa del resultado de explotación, dada la inclusión de los resultados no recurrentes.

Estos y otros estudios han aportado evidencia empírica en empresas ubicadas principalmente en países de Europa, Asia y Norte América. No obstante, se debe señalar que un único conjunto de estándares puede no ser adecuado para todos los entornos y, por lo tanto, 
puede no mejorar de manera uniforme la calidad de la información, especialmente dadas las diferencias entre los países (Soderstrom \& Sun, 2007). De allí que se requiera estudiar otros contextos, como es el caso de empresas ubicadas en economías emergentes (Eng, Lin \& Neiva, 2019), para poder determinar los efectos que ha traído consigo la aplicación de las NIIF, más aún, cuando es esencial para el desarrollo de este tipo de economías, el reducir la opacidad en las ganancias y promover una mayor confianza en la información divulgada (Mongrut \& Winkelried, 2019).

Colombia es un referente que puede servir en este sentido, ya que ha sido uno de los países que ha atendido en los últimos años el proceso de convergencia contable y ha definido la aplicación obligatoria de las NIIF en los estados financieros oficiales desde el año 2015. Esto ha favorecido el reconocer los más recientes lineamientos que en esta materia ha emitido el International Accounting Standards Board (IASB).

En Colombia los estudios sobre esta materia se han enfocado en evaluar la conveniencia de la adopción de las NIIF (Mantilla, 2002; Consejo Técnico de la Contaduría Pública, 2009; Grajales, Cuevas \& Usme, 2015) y de los impactos financieros y patrimoniales en su aplicación (Lasso, Vargas \& Ruano, 2018; Superintendencia de Sociedades, 2015). Se ha señalado que una vez adoptadas las NIIF se logran cifras contables más ajustadas a una realidad financiera, trascendiendo las necesidades fiscales que determinaban la forma de generar la información contable en el país. De igual forma, se ha evidenciado una disminución en las cifras patrimoniales de un número significativo de empresas, una vez, se ha aplicado el nuevo marco contable.

En este sentido, los estudios realizados se han enfocado en estudiar la relevancia valorativa de las NIIF, dejando en un segundo plano, el estudio empírico de la relevancia informativa en términos de observar la variación en las prácticas de revelación de información. Para Holthausen y Watts (2001) la variedad de demandas de informes financieros de partes distintas de los inversores bursátiles hace que las pruebas de relevancia de valor pueden ser menos relevantes para el objetivo de los emisores de normas y los objetivos de los informes financieros.

Este tema, aunque no aparece en la agenda de muchos investigadores, no deja de ser menor en el estudio de la regulación contable, ya que puede entenderse como un tema que pasa por las preocupaciones políticas al interior de las organizaciones para decidir qué revelar, lo cual es quizás distinto a "los problemas de medición" que en gran medida son un tema técnico y no político (Coy, Fischer \& Gordon, 2001).

Tanto los investigadores como los profesionales contables saben que los estados financieros no se explican por sí mismos. En consecuencia, las descripciones y explicaciones que se logran por medio de las notas a los estados financieros son cruciales para contextualizar y comprender la información proporcionada. Por lo tanto, si las compañías no dimensionan los elementos narrativos para complementar la información cuantitativa, existe el riesgo de 
reportar un conjunto de estados financieros que no logren cumplir con su objetivo último, el cual es el suministrar información relevante y útil para la toma de decisiones económicas (Henderson, 2019).

A demás de analizar los efectos de la adopción obligatoria de las NIIF en las prácticas de relevación, es importante considerar si existen factores asociados a las características de las empresas que hayan sido impulsores de la adopción y, en este caso, del nivel de revelación de información lograda después de la adopción de las NIIF. Autores como Mongrut y Winkelried (2019) y Hutagaol, Valentincic y Warganegara (2019) consideran que la mera adopción de las NIIF no aumenta, por sí sola, la calidad del proceso de información financiera y, por ende, no garantiza la transparencia en los mercados emergentes.

A partir de lo anterior, el objetivo del presente estudio es analizar el impacto de la aplicación de las NIIF en las prácticas contables de revelación de información en empresas colombianas. Para ello, se realiza un análisis de contenido en las notas a los estados financieros de los años 2014 y 2015 en 31 empresas cotizadas pertenecientes al sector industrial colombiano. Con esto se busca entender si ha existido o no un beneficio en términos de una mayor relevancia informativa. De igual forma, se evalúa si el grado de revelación obtenido con la aplicación de las NIIF puede estar determinado, más allá de su aplicación obligatoria, por variables empresariales que puedan explicar la mayor revelación de información.

El artículo inicia con la introducción expuesta y avanza en un marco teórico que expone las presiones que la nueva regulación y las características de las empresas pueden tener en la mayor revelación de la información contable. Luego se llega a la metodología que sustenta el trabajo empírico. A partir de esto, se presentan los resultados y las conclusiones sobre el impacto de la aplicación de las NIIF en materia de revelación de la información.

\section{Marco teórico}

La revelación de información se configura en un mecanismo deseado de transparencia organizacional que favorece la toma de decisiones por parte de los grupos de interés (Schnackenberg \& Tomlinson, 2016; Henderson, 2019). La literatura contable ha hecho uso de distintas teorías para comprender el papel y la relevancia que tiene la mayor revelación de información en las prácticas corporativas (García \& Sánchez, 2006).

Se han planteado distintas posturas desde teorías como: la de la Agencia, la de las Señales, la de los Costes del Propietario, la de los Costes de Transacción e Institucional (Hoque, 2006, Hellman, Carenys \& Moya, 2018). Estas teorías plantean que la revelación de información puede servir: a). como una forma de acercar al principal y al agente, con el fin de mitigar las asimetrías de información y permitir un mejor control, b). favorecer las relaciones entre las organizaciones y sus distintos grupos de interés dada la influencia que puede existir entre 
unos y otros, y c). como un mecanismo para señalar al mercado la mejor posición relativa de una empresa frente a las demás (García \& Sánchez, 2006).

Bajo estas teorías, la selección de las políticas frente a la información organizacional a revelar dependerá de las exigencias normativas y de la voluntad por generar más información, las cuales van a depender de las percepciones que los directivos tengan sobre sus costos y beneficios (Albu, Albu \& Gray, 2020; Hellman, Carenys \& Moya, 2018), y de las influencias que se originan por el complejo conjunto de fuerzas de oferta y demanda informativa (Larran \& García, 2004).

En este sentido, la generación de información contable está sujeta a dos influencias diferentes: la presión de los organismos reguladores y las presiones del mercado (Giner, 1997). La primera, considera que la calidad de la contabilidad es una función del entorno institucional, incluido el sistema legal y político del país en el que reside la empresa (Soderstrom \& Sun, 2007). Se cree que el entorno regulatorio en el que operan las empresas influye en la calidad informativa de los informes financieros divulgados, ya que al monitorear la información, los organismos reguladores aumentan los incentivos para la elaboración de informes financieros de alta calidad (Firmino, Nogueira, Cavalcante, Magalhães \& Rodrigues, 2019).

Esta influencia hoy en día comprende los procesos de convergencia contable hacia estándares internacionales de información financiera, los cuales se han adoptado ampliamente en todo el mundo (El-Helaly, Ntim \& Al-Gazzar, 2020). Esto ha llevado a que tanto reguladores como los preparadores de la información reconozcan nuevos criterios y revisen los ya existentes para poder lograr una información que atienda las nuevas demandas informativas que surgen desde las disposiciones internacionales.

La segunda presión surge del mercado, lo cual corresponde a los aspectos y situaciones que condicionan el actuar de las empresas. Esto se refleja en las características que se encuentran asociadas con los atributos de las compañías (Giner, 1997). De allí que se haya querido comprobar cómo estas características son factores que pueden determinar el nivel de información que revelan las empresas (Hellman, Carenys \& Moya, 2018). Esto permite entender que la revelación de nueva información puede trascender el papel vinculante de las normas y considerar otros factores explicativos (Mongrut \& Winkelried, 2019; Hutagaol, Valentincic \& Warganegara, 2019).

Lo anterior tiene sentido si se considera que se ha evidenciado que las mejoras en la calidad contable después de la adopción de las NIIF se limitan a las empresas con incentivos para adoptar, es decir, que los mejores resultados se han obtenido por empresas que han aplicado voluntariamente las NIIF. Christensen, Lee, Walker y Zeng (2015) encuentran que, para el caso de Alemania, aquellas empresas que adoptaron voluntariamente dichos lineamientos, previo a su obligatoriedad en el año 2005, lograron mejores condiciones en la calidad de la información. 
Soderstrom y Sun (2007) han planteado que el uso de un solo conjunto de estándares de contabilidad puede no mejorar la calidad de la contabilidad de manera uniforme para cada empresa y país debido a factores adicionales. Por ende, resulta necesario entender el impacto que genera el adoptar nuevas normas en la revelación de la información y de los factores que caracterizan a las empresas para lograr mejores condiciones de transparencia informativa ante sus grupos de interés.

\section{La presión de la regulación contable en la revelación de información}

Los estudios en esta materia se han enfocado en los últimos años en analizar los efectos de la aplicación de las NIIF en la calidad de la información contable (Firmino, Nogueira, Cavalcante, Magalhães \& Rodrigues, 2019; Cardona, Gómez \& Cano, 2019; Key \& Kim, 2020). Esta última, asumida desde la manipulación del resultado y el reconocimiento oportuno de pérdidas, como atributos contables o desde la relevancia valorativa e informativa, como atributos de mercado (Christensen, Lee, Walker \& Zeng, 2015; Hellman, Carenys \& Moya, 2018; Cardona, Gómez \& Cano, 2019; Eng, Lin \& Neiva, 2019).

Frente a esto último, Soderstrom y Sun (2007) y Angla (2013) señalan que el estudio sobre la calidad de la información contable se ha planteado desde la perspectiva de los inversores del mercado de valores, ya que se consideran que tomar decisiones sobre cuándo y cuánto invertir o desinvertir, se hace preferiblemente a partir de disponer de más información para reducir la incertidumbre relacionada con la rentabilidad y los riesgos futuros, por lo que la existencia de poca información puede llevar a decisiones sesgadas. Se supone que, a más información mayor calidad y viceversa (Giner, 1995; Angla, 2013). Esto es un reflejo de uno de los requisitos exigidos en el marco conceptual de las NIIF para asegurar la calidad de la información contable a través de la información que se revela.

Para el IASB los estados financieros deben satisfacer la cualidad de la relevancia, es decir, que la información incorporada en estos debe ser capaz de influir en las decisiones de los usuarios (International Accounting Standards Board, 2010). Los datos expuestos en los estados financieros y la información en sus notas deben favorecer la comunicación eficaz, su adecuada comprensión y evitar el exceso de información que trae consigo costos en el tiempo para poderla analizar (International Accounting Standards Board, 2017a).

Las revelaciones por medio de las notas a los estados financieros, al proporcionar información sobre las políticas, estimaciones, juicios contables y explicación de los hechos económicos, permiten aclarar la situación y resultados financieros que la administración muestra por medio de los estados financieros. Esto posibilita una mejor información que lleve a una toma decisiones informadas y a una mejor evaluación del desempeño de la empresa. 
Bajo lo anterior, se entiende que la revelación de la información es un término amplio que hace referencia a la responsabilidad frente a otras personas o partes de interés (Mantilla, 1996), por lo que las empresas no pueden ignorar el cumplimiento de unos mínimos requerimientos a revelar sobre las partidas contables (Hellman, Carenys \& Moya, 2018).

Si bien la información a divulgar depende del juicio de su preparador, al decidir qué información revelar en los estados financieros y la forma más eficaz de organizarla y comunicarla, se hace necesario la observancia de los requerimientos que traen las normas contables para exponer aquellos aspectos que como mínimo se proponen y que se entienden son relevantes para los usuarios (Henderson, 2019).

Así, estudios como el desarrollado por Barth, Landsman y Lang (2008), en 327 empresas ubicadas en 21 países de Europa, Asía y África, encontraron que aquellas compañías que siguieron los lineamientos de las NIIF lograron una mejor calidad en la información contable, que aquellas que siguieron los lineamientos locales. Iatridis (2010), al evaluar el cambio de los PCGA del Reino Unido a las NIIF, encontró que existe una reducción en la manipulación de los resultados, dado el reconocimiento más oportuno de pérdidas y la generación de medidas de valor más relevantes, por lo que este autor concluye que las NIIF han permitido una menor asimetría y mayor calidad de la información contable que favorece a los inversionistas para hacer juicios informados e imparciales.

A su vez, Harakeh, Lee y Walker (2019) al examinar el potencial de las NIIF para influir en el mercado de acciones en el Reino Unido y en Francia, encuentran que, aunque existe una divergencia menor entre las normas nacionales de contabilidad del Reino Unido y las NIIF (empresas de baja divergencia), y una divergencia material de las normas nacionales de contabilidad en Francia frente a las NIIF (empresas de alta divergencia), la adopción de dichas normas ha servido para mitigar la asimetría de la información y mejorar la calidad de la contabilidad.

Al evaluar otros contextos, Key y Kim (2020), encuentran en 439 empresas de Corea del Sur un fuerte compromiso con una implementación exitosa y transparente de las NIIF, lo cual ha incidido positivamente en la calidad contable. Los resultados son en gran medida consistentes con una menor administración de ganancias luego de la adopción de las NIIF y un reconocimiento de pérdidas más oportuno, lo que indica una mayor calidad contable.

En este mismo sentido, Dayanandan, Donker, Ivanof y Karahan (2016) determinaron la existencia de cambios en la información financiera después de la aprobación de las NIIF en Europa y en países ubicados en los demás continentes. Para ello, evaluaron la información de 3030 empresas de 35 países y evidenciaron la suavización de los ingresos y la gestión de las ganancias. El estudio concluye que la gestión de las ganancias ha disminuido en el período posterior a las NIIF, en particular, para los países de derecho civil francés y escandinavo, pero no para los países de derecho civil alemán y los países de derecho consuetudinario. Esto 
último se puede explicar por el hecho de que los países de common law tienen leyes sólidas de protección de los inversores, estricto cumplimiento de la ley y altos niveles de revelación de información financiera. En general, el estudio muestra que la adopción de las NIIF mejoró la calidad de los informes financieros.

De Moura, Altuwaijri \& Gupta (2020) investigaron si la adopción de las NIIF ha afectado el costo del capital de largo plazo y la deuda en empresas de América Latina, donde la aplicación de las normas contables y los mecanismos de protección de los inversores son débiles en comparación con las naciones desarrolladas. Analizando una muestra de empresas de Argentina, Brasil, Chile, México y Perú, demostraron que la adopción obligatoria de las NIIF condujo a una reducción en el costo de capital. Los resultados sugieren que la divulgación mejorada y la comparabilidad derivadas de las NIIF en comparación con las normas contables nacionales anteriores ayudaron a mitigar el problema de asimetría de la información y tuvieron consecuencias económicas positivas para las empresas latinoamericanas.

Así, las NIIF han contribuido en mejorar cualidades de la información financiera suministrada, con lo que se espera se generen oportunidades y ventajas en materia de transparencia informativa para las empresas. Carreño (2015) señala ventajas que se derivan de la aplicación de las NIIF: 1. El fomentar reformas en los sistemas de reporte gerencial internos para manejar adecuadamente la contabilidad financiera y la generación de estados financieros dentro de la nueva regulación. 2. Perfecciona las medidas para evaluar la compañía y el desempeño de los ejecutivos, enfocándose particularmente en la generación de valor para el accionista, y 3 . Mejora la comunicación de la posición financiera y de los resultados de la compañía, junto con otros indicadores de desempeño, para los analistas, inversionistas y otros usuarios de la información financiera.

Sin embargo, Grossman, Smith y Tervo (2013), al comparar en Estados Unidos la presentación de los estados financieros bajo las NIIF, con respecto a los obtenidos bajo los US GAAP en 126 empresas de la Unión Europea que cotizan en la bolsa de New York, encontraron que no existen diferencias significativas en los resultados de los estados financieros y que la información expuesta es muy similar, por lo que las normas internacionales en países como Estados Unidos no han causado dificultad al momento de considerar su uso.

Cardona, Gómez y Cano (2019) muestran que en compañías cotizadas en Latinoamérica y el Caribe durante los periodos 2006-2016, se ha dado un mejoramiento parcial en la calidad contable, evidenciado a través de una disminución en el nivel de manipulación del resultado sólo después de varios años de aplicación de las NIIF. Sin embargo, no se evidencia mayor oportunidad al reconocer grandes pérdidas, al tiempo que, la información contable generada bajo las NIIF no es valorada con mayor utilidad por los agentes de mercado.

Por su parte, Abdul y Haniffa (2020) encuentran que, la adopción de las NIIF en Nigeria después de que se fortaleció la aplicación de las normas contables, han tenido una afectación 
negativamente en la calidad de la contabilidad, ya que mientras que la gestión de ganancias aumentó, el reconocimiento de pérdidas oportunas y la persistencia de ganancias se redujeron.

En este sentido, los estudios en su conjunto demuestran que el efecto de la adopción de las NIIF es contextual, es decir, que no se puede señalar que la mejora de la calidad de contabilidad o de la información en términos de su relevancia valorativa o informativa depende solo de la adopción de las NIIF y que su efecto es el mismo para todos los países, por lo que el reconocer las presiones que existen del mercado es un asunto complementario en la investigación contable.

\section{La presión del mercado en la revelación de información}

Para analizar la forma como se evidencia esta presión, la investigación contable ha analizado la presencia de distintos factores que pueden afectar las prácticas informativas de las empresas. Para ello, se ha señalado la existencia de características empresariales que pueden incidir en el nivel de información que se reporta (Hellman, Carenys \& Moya, 2018).

Soderstrom y Sun (2007) indican que la elección de las normas de contabilidad de una empresa debe predecirse por factores, tales como: el origen legal, el nivel de endeudamiento, la propiedad, el ciclo operativo, la exposición internacional y el tipo de industria, entre otros. De Lima, De Lima, De Carvalho y Lima (2010) han encontrado para el caso de empresas brasileras que los incentivos a nivel de empresa son importantes impulsores del cumplimiento de las prácticas de convergencia de las NIIF. Los resultados sugieren que las empresas que son más grandes, que están más expuestas a los mercados internacionales y tienen mayores necesidades de financiamiento, son más proclives a adoptar prácticas NIIF implementando cambios sustanciales en sus políticas contables.

Para García y Sánchez (2006) el tamaño de la empresa, la cotización bursátil, la concentración de la propiedad, el tamaño del auditor, el nivel de endeudamiento o la rentabilidad son algunas de las variables que de alguna manera se han asociado con la política de revelación de información de las empresas. Para estos autores las empresas son conscientes de los beneficios que conlleva la oferta de una mayor cantidad de información, entre los que destacan la reducción del coste de capital, mejora de la liquidez e imagen, beneficios en la gestión interna, reducción de la asimetría informativa, aumento de la cobertura de analistas, credibilidad y reputación de la compañía y beneficios colectivos derivados de la mejora en el crecimiento económico, empleo y nivel de vida.

Por otra parte, Mir, Moreno y Olmeda (2006) han estudiado, en el ámbito de las empresas españolas, como las diferentes propuestas normativas que se han ido desarrollando en los últimos años en torno a la información contable sobre derivados financieros, con la presencia cada vez más próxima de las proposiciones del IASB, tuvieron una influencia significativa en 
la transparencia y calidad de los datos contables reportados por las entidades cotizadas. El trabajo a partir de la evolución normativa de los años 2000 a 2002 evalúo la generación de información voluntaria sobre derivados financieros. En función de ello, los autores, además, concluyen que existen determinantes que inciden en la revelación de información sobre derivados financieros, los cuales se vinculan con el tamaño de la empresa y el volumen de endeudamiento.

Zheng y Chen (2017) consideran que la calidad en el reporte financiero de las empresas en China se encuentra condicionada por la industria, la ubicación geográfica y la tasa de rendimiento (ROE) en el capital contable de las empresas. No obstante, muestra que la auditoría externa tiene una influencia significativa en la calidad de los informes financieros, pero sin que existan resultados concluyentes, por lo que es un factor que aún debe ser estudiado.

En estudios recientes, Mnif y Znazen (2020) consideran, en el ámbito de las empresas canadienses, el efecto de dos mecanismos de gobierno corporativo: el consejo de administración y el comité de auditoría, en el nivel de cumplimiento de las divulgaciones obligatorias de la NIIF 7 "Instrumentos financieros: información a revelar". Encuentran que el nivel de cumplimiento tiene un vínculo positivo con el tamaño del Consejo y la independencia de sus miembros. De manera similar, se demuestra que la independencia del Comité de Auditoria y la experiencia en contabilidad financiera afectan positivamente el cumplimiento de la NIIF 7. Sin embargo, la dualidad CEO / presidente, el tamaño del Comité de Auditoria y la frecuencia de las reuniones no se correlacionan significativamente con el nivel de cumplimiento con la NIIF 7.

Tawiah y Boolaky (2019) al examinar los impulsores del cumplimiento de las NIIF en 205 empresas en 13 países africanos, encuentran que existe una relación positiva y significativa entre el cumplimiento y el comité de auditoría. En contraste, hay una relación inversa entre el nivel de cumplimiento y la concentración de la propiedad. Estos mismos autores estudiaron los determinantes de la variación en los ajustes de capital entre las empresas indias a partir del proceso de convergencia a las NIIF (Tawiah \& Boolaky, 2020). Con una muestra de 323 empresas que cotizan en bolsa, demostraron que el monto del ajuste total de capital está sujeto a las características de la empresa, como son el apalancamiento, el nivel de control familiar, el tipo de auditor y la estructura de propiedad.

Es así como los resultados obtenidos en la investigación empírica sobre los efectos en la calidad de la información, a partir de la adopción de las NIIF, deben interpretarse en relación, no solo de las presiones que ejerce la regulación y las instituciones, sino también a la luz de factores específicos del país y de las empresas (Pascan, 2015; Hellman, Carenys \& Moya, 2018). 


\section{Hipótesis del estudio}

A partir de la evidencia obtenida sobre el efecto que ha tenido las NIIF sobre la calidad de la información en empresas ubicadas en contextos distintos al colombiano, se busca evidenciar si ha existido el mismo efecto en las prácticas contables seguidas en las empresas colombianas. En este sentido, se formula la primera hipótesis del estudio.

H1: ha existido una mejoría en la calidad de la información revelada por parte de empresas colombianas una vez se han aplicado las NIIF.

Por otra parte, al considerar que los efectos en la revelación de la información pueden ser consecuencia de presiones de mercado, las cuales se reflejan en las características de las empresas, trascendiendo las presiones regulativas, se consideran como posibles factores explicativos para la presente investigación: el tamaño de la empresa, su nivel de endeudamiento, su rentabilidad, la calidad del auditor, su grado de internacionalización y su edad.

Al respecto, la evidencia empírica ha demostrado resultados heterogéneos, por lo que en la actualidad no existe una teoría general que permita explicar o predecir el comportamiento de la empresa en relación con la revelación de información (García \& Sánchez, 2006; Hellman, Carenys \& Moya, 2018). Se han asumido un conjunto de teorías parciales para comprender la cantidad de información divulgada. En particular, se han planteado posturas desde teorías, como: la de la Agencia, la de las Señales, la de los Costes del Propietario, la de los Costes de Transacción y la de la Eficiencia de los Mercados (Hellman, Carenys \& Moya, 2018; Nassreddine, 2016; Angla, 2013; Bravo, Abad \& Trombetta, 2009; Larrán \& García, 2004). Para la presente investigación se toma como referente la teoría de la agencia para justificar la posible relación entre los factores determinantes y la cantidad de información revelada.

\section{Tamaño de la firma}

Con base en García y Sánchez (2006) se considera la teoría de la Agencia como un sustento teórico razonable para entender la relación entre tamaño y revelación de información, ya que esta permite comprender que cuanto mayor sea el tamaño de una organización, más importancia tendrán los potenciales conflictos de intereses y por tanto mayores serán los costes de agencia entre accionistas y directivos. Se considera, entonces que, a medida que aumenta el tamaño de una empresa, existe un mayor interés por la compañía y, probablemente, una mayor demanda de información sobre esta (Larrán \& García, 2004).

Investigaciones previas han determinado la existencia de una relación significativa entre esta variable y la revelación de información (Hellman, Carenys \& Moya, 2018; De Lima, De Lima, De Carvalho \& Lima, 2010; Bonsón \& Escobar, 2004) sugiriendo que las grandes compañías suelen llevar mejores y más amplias políticas de revelación de información. No 
obstante, autores como Aksu y Kosedag (2006) no encuentran relación entre estas variables, por lo que consideran que probablemente el hecho de existir información obligatoria hace que la variación en la revelación de información sea menor. Por lo tanto, la hipótesis que se formula es la siguiente:

H2: la revelación de información contable en empresas colombianas, a partir de la aplicación de las NIIF, se encuentra positivamente relacionada con el tamaño de la empresa.

\section{Nivel de endeudamiento}

La teoría de la Agencia afirma que se espera una mayor transferencia de riqueza de los acreedores a los accionistas conforme el nivel de endeudamiento sea mayor, ya que los costes de agencia son más altos para empresas que utilizan más financiación ajena (García \& Sánchez, 2006). En este sentido, se espera que una mayor divulgación de información a las agencias de calificación y a los prestamistas reduzca el costo de la deuda (Armitage \& Marston, 2008). Mi, Sheng y Elrod (2016) consideran que una empresa va a mejorar su calidad de divulgación de información cuando se decide financiar, ya que la calidad de la divulgación de información puede ser vista como su reputación. De allí que los resultados empíricos muestran una correlación positiva entre las decisiones de la divulgación de información y la financiación (Tawiah \& Boolaky, 2020).

H3: la revelación de información contable en empresas colombianas, a partir de la aplicación de las NIIF, se encuentra positivamente relacionada con el nivel de endeudamiento de las empresas.

\section{Rentabilidad}

La cantidad de información divulgada puede estar relacionada con la variabilidad de la rentabilidad de la empresa por las asimetrías informativas generadas y la mayor posibilidad de litigios debido a su mayor volatilidad en precios (García \& Sánchez, 2006; Hellman, Carenys \& Moya, 2018). Desde la perspectiva de la teoría de la agencia, cuando la rentabilidad de la empresa es buena, los directivos desearán hacerlo saber a los propietarios para mejorar la imagen de la empresa y su nivel de remuneración (Subramaniam, 2006). Por lo tanto, resulta necesario evidenciar la relación existente entre la información revelada y el desempeño financiero de la empresa.

H4: la revelación de información contable en empresas colombianas, a partir de la aplicación de las NIIF, se encuentra positivamente relacionada con el nivel de rentabilidad de las empresas. 


\section{Calidad del auditor}

Desde la teoría de agencia se ha señalado que los gerentes sabiendo que los accionistas buscarán controlar su comportamiento a través de las actividades de vinculación y monitoreo (Subramaniam, 2006), divulgarán más información para convencer a los accionistas de que están actuando de manera óptima. Asumiendo que la calidad del auditor se puede medir en términos de su tamaño, se considera que el hecho de estar auditadas las cifras contables por alguna de las cuatro firmas de auditoría más representativas en el mundo (Big Four), se obtienen ciertas condiciones deseadas de experiencia y de experticia para la generación de una información con mejores condiciones en materia de revelación (Mnif \& Znazen, 2020; Albu, Albu \& Gray, 2020), por lo se espera que las grandes firmas de auditoría estén más preocupadas por lo que sus clientes divulgan.

Varios estudios han analizado empíricamente la relación entre el tamaño del auditor y el grado de revelación de la información (Agyei, 2017; Ahmed \& Karim, 2005), así como con el cumplimiento de las NIIF (Tawiah \& Boolaky, 2019). Se ha determinado que esta variable ejerce una influencia significativa sobre el volumen de información revelado al incrementar la preocupación de la empresa por el diseño de una política informativa de calidad (García \& Sánchez, 2006).

No obstante, Mongrut y Winkelried (2019) han sugerido que la calidad de la auditoría solo importa como una señal sustitutiva para las empresas que no han adoptado las NIIF, ya que han evidenciado que existen empresas en donde la adoptación de las NIIF se asocia con una menor calidad de auditoría, por lo que los gerentes podrían creer que la simple adopción de las normas internacionales es suficiente para garantizar una mayor transparencia. Por ende, la relación a estudiar es:

H5: la revelación de información contable en empresas colombianas, a partir de la aplicación de las NIIF, se encuentra positivamente relacionada con la calidad del auditor de la empresa.

\section{Grado de internacionalización}

Las empresas que operan en un escenario internacional tienden a revelar mayor cantidad de información en aras de captar clientes internacionales, capital extranjero a un menor coste y mejorar su imagen pública (García \& Sánchez, 2006). A su vez, el entorno empresarial de las empresas que participan en contextos internacionales es más complejo que el de las empresas nacionales, por lo que la existencia de regulaciones uniformes para la elaboración y presentación de estados financieros benefician no solo a los inversores, acreedores, analistas financieros sino también a los procesos como la contabilidad y la auditoría (Tran, Ngo, Phan, Do $\&$ Pham, 2020) y facilita la comparación de los estados financieros en empresas de diferentes 
países, a partir de la información que se revela (Rico, Montoya, Franco \& Laverde, 2020).

Trabajos previos ponen de manifiesto relaciones positivas entre el nivel de ventas extranjeras y los niveles de revelación (Skouloudis, Jones \& Malesios, 2014; De Lima, De Lima, De Carvalho \& Lima, 2010). Cooke (1989) enfatiza que la presencia de una empresa en mercados foráneos, le obligada a divulgar información más completa de acuerdo con las reglas de información del sistema de negocios extranjeros. Así, la relación a plantear es:

H6: la revelación de información contable en empresas colombianas, a partir de la aplicación de las NIIF, se encuentra positivamente relacionada con el nivel de internacionalización de la empresa.

\section{Edad de la firma}

La edad de una empresa se ha considerado a partir del tiempo transcurrido, una vez ha sido incluida en un mercado público de valores (Zeng, Xu, Yin \& Tam, 2012; Liu \& Anbumozhi, 2009). Se espera que una empresa que ha estado en el mercado por más tiempo ha consolidado mejor su estructura de control y presentación de informes (Coluccia, D’ Amico, Fontana \& Solimene, 2017), lo que les permite dar cuenta de una mejor y mayor cantidad de información a revelar.

Por su parte, Bravo (2016) y Zeng, Xu, Yin y Tam (2012), demuestran que la edad tiene una relación inversa con el nivel en que las empresas divulgan su información. Esto puede indicar que el tiempo que lleve una empresa en el mercado, tiene baja incidencia en el nivel de revelación de información a terceros, o que las empresas jóvenes buscan capturar la atención y lograr presencia en el mercado dando a conocer de manera amplia su desempeño para lograr credibilidad y confianza ante sus grupos de interés. Esto último puede tener sentido bajo lo expuesto en la teoría de la señalización (Hoque, 2006). Las empresas pueden estar interesadas en proporcionar información como una señal o como un mecanismo para proveer al mercado información adicional sobre sus circunstancias financieras, con el fin de cambiar las expectativas de los inversores y reducir las asimetrías de información (Frías, Rodriguez \& García, 2014). Por lo tanto, se espera que:

H7: la revelación de información contable en empresas colombianas, a partir de la aplicación de las NIIF, se encuentra positivamente relacionada con la edad de la empresa. 


\section{Diseño de la investigación}

\section{Selección de la muestra}

La muestra seleccionada corresponde a las empresas cotizadas en Colombia pertenecientes al sector industrial colombiano a febrero de 2017. En total se encontraron 67 empresas, de las cuales se seleccionaron las que reportaban algún grado de capitalización bursátil y que para los años 2014 y 2015 reportaban en sus estados financieros partidas asociadas con los inventarios y la propiedad, planta y equipo. A partir de estos criterios, se obtuvo como resultado un total de 31 compañías que cumplen con estas condiciones.

El estudiar la revelación de información en empresas pertenecientes a un sector o segmento especifico, corresponde a la idea de la existencia de sectores económicos con características particulares que pueden afectar las prácticas contables al momento de evaluar los cambios normativos que se incorporan en la legislación local. Esto lo evidenció Bauman (2013) al examinar las diferencias significativas en los requerimientos de revelación de activos fijos bajo los PCGA anteriores y actuales y bajo NIIF en Estados Unidos. Antes de 1994 la SEC requería que las empresas que cotizaban en bolsa y con capital intensivo prepararan información complementaria que detallara la actividad de las cuentas de los activos fijos. Si bien este requisito de presentación de informes se eliminó en los Estados Unidos, las empresas que informaron bajo NIIF estuvieron sujetas a requisitos de divulgación similares a los requeridos por la SEC antes de los ajustes a la normativa local, por lo que las NIIF favorecieron la revelación de una mayor información en esta partida contable, en comparación con lo exigido para este segmento económico por parte de las actuales normas locales.

Asimismo, es de considerar que la composición económica de Colombia se caracteriza por tener participación relevante de los sectores financiero e industrial. De cada \$1 000 que se crean en el país, aproximadamente \$200 lo aportaron las instituciones financieras, \$100 el sector industrial y $\$ 60$ el sector agropecuario (¿Cómo está compuesta la economía colombiana?, 2015). De igual forma, el Departamento Administrativo Nacional de Estadística (DANE, 2017), mostró que entre los sectores económicos con mayor crecimiento para el año 2016 se encontraba el sector industrial, el cual logro un 3\% de crecimiento, siendo este superior al general $(2.0 \%)$ expuesto por toda la economía colombiana.

Estas empresas al estar inscritas en el registro nacional de valores deben aplicar como nuevo cuerpo normativo las NIIF emitidas por el IASB. De estas empresas, se obtuvieron sus estados financieros con corte a 31 de diciembre de 2014 y 2015, es decir, los informes oficiales antes y después de la aplicación del nuevo cuerpo normativo contable para realizar una comparación entre periodos y, con ello, evidenciar los cambios que ha traído la aplicación de la nueva regulación. Esta forma de comparación ha sido aplicada por Barth, Landsman y 
Lang (2008) para poder determinar si el uso de nuevos lineamientos ha tenido incidencia o impacto en mejores prácticas contables en las empresas después de su aplicación.

Si bien para el año 2014 las NIIF no eran de obligatoria aplicación para preparar los estados financieros oficiales, puede ocurrir que en el proceso de implementación que iniciaron estas empresas se haya decidido revelar voluntariamente algunos asuntos que resultaran aplicables para la situación y resultados financieros reportados. Esta práctica puede ser una señal a sus grupos de interés sobre la generación de una información con mejores condiciones de revelación y demostrar su interés por ir atendiendo las exigencias de la nueva regulación (Christensen, Lee, Walker \& Zeng, 2015). Por ende, se asume como parámetro de comparación para los dos periodos los requerimientos de revelación previstos en las NIIF.

Este tipo de análisis ha sido desarrollado en el contexto colombiano por Católico, Cely y Pulido (2013), quienes previo a la adopción obligatoria de las NIIF, evaluaron el grado de revelación de la información sobre propiedad, planta y equipo de 59 empresas industriales que cotizaban en la Bolsa de Valores de Colombia. Frente a los cambios en los requerimientos de la información que potencialmente podría traer consigo las NIIF, encontraron que solo una de las compañías superaba el $50 \%$ de los requerimientos de información en sus notas a los estados financieros y que las 58 empresas restantes tenían un grado de revelación entre el $48 \%$ y $22 \%$, lo cual demostraba resultados desfavorables. Por lo tanto, se esperaría que una vez hayan iniciado formalmente los procesos de implementación de las NIIF se diera una amplitud en la información revelada.

\section{Variables y métodos de comprobación de las hipótesis}

\section{Variable dependiente}

Para resolver H1 se considera un enfoque descriptivo de las prácticas de divulgación de información. Para ello, se diseñó un índice de revelación $(I R)$ no ponderado, tal como se ha utilizado en investigaciones previas (Mnif \& Znazen, 2020; Hellman, Carenys \& Moya, 2018; Ioana, Tirón \& Pali, 2014; Bravo, Abad \& Trombetta, 2009), a partir de 37 requerimientos de revelación de información que exponen las NIIF en materia de inventarios (NIC 2) y propiedad, planta y equipo (NIC 16). La información sobre el cumplimiento de los requerimientos de revelación se obtuvo a partir de un análisis de contenido de las notas de los estados financieros de las empresas seleccionadas para los años 2014 y 2015. La formalización de este índice se muestra a continuación.

$$
I R=\sum_{1}^{n} A i / n
$$


Donde $A i$ son los requerimientos efectivamente revelados y "n" es el número total de requerimientos que se esperan sean revelados, que para el caso de inventarios es $=10 \mathrm{y}$ propiedad, planta y equipo $=27$, para un total de 37 requerimientos .

Se estudian de manera específica estas dos partidas contables, ya que la importancia relativa que tienen por su naturaleza y cuantía en este tipo de empresas hace de estas los principales activos con los que operan. Para el año 2014, los inventarios en promedio representan, para las empresas analizadas, el $6.73 \%$ y la propiedad, planta y equipo el $43.71 \%$ del total de sus activos. Para el 2015, esta participación fue del $8.13 \%$ y $43.57 \%$, respectivamente.

Adicional, se ha detectado que una vez se han aplicado las NIIF estas partidas muestran variaciones significativas en cuanto a su valor. La Superintendencia de Sociedades $(2014,2015)$ evidenció que, tanto al momento de aplicar por primera vez las NIIF en empresas cotizadas de Colombia, como un año después, es decir, a 01 de enero de 2014 y al cierre del mismo año, el mayor impacto en sus activos estuvo representado por un incremento en la propiedad, planta y equipo, como consecuencia de la aplicación del valor revaluado como costo atribuido.

Esto fue ratificado por Lasso, Vargas y Ruano (2018) quienes observaron para las PyMes de la industria manufacturera en Colombia un incremento patrimonial, en términos generales, y de manera particular, para los inventarios reducciones en su valor, mientras que, en la propiedad, planta y equipo, se hallaron incrementos significativos. Por lo anterior, se esperaría que ante estas variaciones se posibilite una mayor revelación de información para que sus grupos de interés encuentren justificación sobre el comportamiento de las cifras contables.

Cada uno de los requerimientos de revelación se califica como una variable dicotómica para su cuantificación (Cooke, 1989; Católico, Cely \& Pulido, 2013). De allí que se asigne una puntuación de 0 cuando no se revela la información correspondiente y 1 cuando se expone completamente. Con la agregación lineal de estas puntuaciones se calcula el $I R$.

\section{Variables independientes}

Entendiendo que no todas las empresas tienen la misma motivación ni incentivos frente a la mejora de la información, se busca identificar qué variables empresariales son las que determinan la mayor revelación de información (hipótesis H2-H7). Para ello, se trabajan las variables justificadas en el marco teórico y se miden de la forma que estudios previos las han definido (tabla 1).

Adicional a estas variables, se considera la inclusión de una variable que permita controlar el efecto directo en el incremento del grado de revelación de la información para el año 2015, como consecuencia de la adopción de las NIIF. Estudios previos han reconocido la necesidad de controlar dicho efecto en los análisis que se han realizado como producto de evaluar los impactos generados en variables financieras o en los procesos de auditoria, una 
vez se han realizado la adopción de las NIIF (Wijayana \& Gray, 2019; Brochet, Jagolinzer \& Riedl, 2013; Griffin, Lont \& Sun, 2009). Estos estudios han medido de manera dicotómica esta variable, para representar la situación de adopción o no de las NIIF. Ante esta forma de medición, se hace una variación, ya que las empresas estudiadas han adoptado plenamente las NIIF, por lo que se considera que lo más adecuado es utilizar, como medida aproximada, el cambio porcentual producido en el grado de revelación de la información entre los años 2014 y 2015 a partir de la adopción de las NIIF (Var_NIIF).

Tabla 1

Variables independientes

\begin{tabular}{ll}
\hline \multicolumn{1}{c}{ Variable } & \multicolumn{1}{c}{ Definición } \\
\hline End: Nivel de endeudamiento (+) & Relación total pasivo/ total activo \\
Rent: Rentabilidad (+) & Uso del indicador ROA \\
CalAudit: Calidad del auditor & $1=$ si el auditor es una de las Big Four; 0= caso contrario \\
Inter: Nivel de internacionalización & $1=$ tiene ventas al exterior; 0= no exporta \\
Edad $(+)$ & Tiempo de existencia como empresa listada en la BVC \\
Tam: Tamaño $(+)$ & Volumen de activos \\
Var_NIIF & Cambio \% en el grado de revelación una vez adoptadas las NIIF \\
\hline
\end{tabular}

Fuente: Elaboración propia

Para medir la relación entre los factores expuestos en cada una de las hipótesis (variables independientes) con el $I R$ (variable dependiente), se plantea un modelo de regresión múltiple por Mínimos Cuadrados Ordinarios (MCO), método que se ha utilizado en estudios previos (Hellman, Carenys \& Moya, 2018; Alonso, 2009; Mir, Moreno \& Olmeda, 2006; Liu \& Anbumozhi, 2009; Braam, Uit, Hauck \& Huijbregts, 2016) y el cual tiene como expresión:

$$
\begin{gathered}
\text { IR = } \beta_{0}+\beta_{1} \text { End }+\beta_{2} \text { Rent }+ \\
+\beta_{3} \text { CalAudit }+\beta_{4} \text { Inter }+\beta_{5} \text { Edad }+\beta_{6} \text { Tam } \\
+\beta_{7} \text { Var_NIIF }+\varepsilon
\end{gathered}
$$

Los datos trabajados son de corte transversal, correspondiente a las cifras financieras con corte a 31 de diciembre de 2015, fecha que coincide con el cierre contable para el cual se reportan las cifras contables oficiales bajo las NIIF, por lo que se relacionan con el IR de 2015 . Los datos de las variables enunciadas se organizan en SPSS versión 22.0 que es el programa estadístico que se utiliza para realizar los diferentes análisis planteados. 


\section{Resultados del estudio}

\section{Análisis descriptivo del IR}

En la tabla 2 se muestran los resultados obtenidos del $I R$ para los años 2014 y 2015 . Estos, a su vez, se muestran desde el resultado parcial para los inventarios y la propiedad, planta y equipo. Los resultados muestran una mejoría en el promedio de la revelación de información contable (IR 2014=0.31; IR 2015=0.41) después de aplicar las NIIF. De manera particular, los inventarios muestran un incremento marginal al pasar de 0.3 en 2014 a 0.36 en el 2015. En el caso de la propiedad, planta y equipo existe una mejoría sustancial en la divulgación de la información $(2014=0.31 ; 2015=0.43)$, por lo que se está en presencia de un escenario de cambio de regulación con implicaciones favorables en materia de revelación para las empresas cotizadas pertenecientes al sector industrial en Colombia.

Tabla 2

Grado de revelación en inventarios y propiedad, planta y equipo 2014-2015

\begin{tabular}{|c|c|c|c|c|c|c|}
\hline \multirow[t]{2}{*}{ Partida contable } & \multicolumn{3}{|c|}{2015} & \multicolumn{3}{|c|}{2014} \\
\hline & $\begin{array}{l}\text { Inventa- } \\
\text { rio }\end{array}$ & $\begin{array}{c}\text { Propiedad, } \\
\text { planta y equipo }\end{array}$ & Total & Inventario & $\begin{array}{c}\text { Propiedad, } \\
\text { planta y equipo }\end{array}$ & Total \\
\hline $\begin{array}{l}\text { Requisitos por } \\
\text { revelar }\end{array}$ & 10 & 27 & 37 & 10 & 27 & 37 \\
\hline Promedio & 3.6 & 11.5 & 15.2 & 3.0 & 8.5 & 11.5 \\
\hline Máximo & 7 & 16 & 20 & 5 & 12 & 15 \\
\hline Mínimo & 0 & 9 & 10 & 0 & 5 & 5 \\
\hline$I R$ & 0.36 & 0.43 & 0.41 & 0.3 & 0.31 & 0.31 \\
\hline
\end{tabular}

Fuente: Elaboración propia

Este resultado coincide con lo expuesto por Iatridis (2010) y Dayanandan, Donker, Ivanof y Karahan (2016) en países europeos y de Norte América, al observar una mejoría en la calidad de la información revelada y una disminución en la asimetría de información. A su vez, este resultado ha sido ratificado Rico, Montoya, Franco y Laverde (2020), al analizar los efectos de la convergencia de las NIIF, en términos de la comparabilidad de la información financiera de las empresas cotizadas en la Bolsa de Valores de Colombia en el corto plazo, puntualmente en el rubro de propiedades, planta y equipo, quienes muestran que se ha generado un aumento relevante en la divulgación de la información financiera bajo las NIIF. 
No obstante, el resultado obtenido en las dos categorías contables es bajo. Al observar lo divulgado por cada empresa, se muestra que ninguna expone la totalidad de requerimientos que trae la norma, es decir, que, aunque se ha mejorado en la información entregada a sus grupos de interés por medio de los estados financieros y sus notas, aún se tiene un margen para ampliar la información. Esto es más evidente si se comparan los resultados con estudios previos. Hellman, Carenys y Moya (2018) concluyen al revisar distintas investigaciones que el nivel de revelación después de adoptar las NIIF, en promedio, es del 65\%, con un límite inferior en promedio del $46 \%$ en países o regiones de mercados emergentes y en desarrollo durante 1996-2013, lo que sugiere que los resultados obtenidos se encuentran lejos de un nivel mínimo razonable de cumplimiento a partir de la experiencia lograda en otros países.

Es de señalar que esto último puede encontrar sustento en lo planteado por el International Accounting Standards Board (2017b), al momento de realizar por parte de las empresas juicios sobre la materialidad y que tienen implicaciones en los lineamientos de reconocimiento, medición, presentación y revelación de la información:

Una entidad no necesita revelar información especificada por una Norma NIIF si la información procedente de esa revelación no es material o no tiene importancia relativa. Este es el caso incluso si la Norma contiene una lista de requerimientos específicos o los describe como "requerimientos mínimos". Por el contrario, la entidad debe considerar si proporcionar información no especificada por las Normas NIIF, si esa información es necesaria para que los usuarios principales comprendan el impacto de transacciones concretas, $u$ otros sucesos y condiciones sobre la situación financiera, rendimiento financiero y flujos de efectivo de la entidad (p. 10).

A su vez, estos resultados se pueden comprender a partir de la falta de incentivos que había en el año 2014 para adoptar voluntariamente las NIIF (Christensen, Lee, Walker \& Zeng, 2015) y la consiguiente mejora de calidad después de la adopción forzosa en el año 2015. Es de considerar que los requerimientos a revelar dispuestos en los PCGA colombianos y que se tuvieron como referencia para preparar los estados financieros del 2014, eran inferiores a los que exponen las NIIF, por lo que resulta razonable que, bajo una lógica de cumplimiento, las empresas no se sintieran obligadas a ampliar sus notas en los estados financieros antes de la adopción de las NIIF.

Asimismo, la aplicación de las NIIF conlleva a la revisión de la información que se venía presentando. Los preparados de la información son más conscientes de lo que se debe explicar y consignar en las notas a los estados financieros ante nuevos lineamientos. Iatridis (2008) plantea que las empresas están inclinadas a revelar más información, con el fin de asegurar a los participantes del mercado que sus políticas contables son consistentes con la 
nueva regulación. Asimismo, la existencia de incentivos para la obtención de financiación por parte de los mercados de capital y de deuda, se ve reflejado en las mayores revelaciones contables que se hacen (García \& Sánchez, 2006).

Finalmente, la forma de aprendizaje de las NIIF incide en la falta de observancia plena de los requerimientos de revelación. La capacitación en Colombia ha privilegiado el estudio sobre el reconocimiento, medición y presentación de la información, dejando a un lado el análisis y aplicación de lo referente a la revelación de la información, por lo que los preparadores de la información contable al no tener el conocimiento pleno de las normas pueden obviar asuntos materiales por revelar.

En este orden de ideas, se acepta $H 1$ en términos de la mejoría en la calidad de la información expuesta después de adoptar las NIIF. No obstante, el no lograr un cumplimiento pleno hace que se tenga un margen para mejorar, lo cual puede resultar en los siguientes años, como consecuencia de la mejor apropiación de las NIIF y de las mismas presiones del regulador y del mercado.

\section{Descriptivos variables independientes y análisis de correlaciones}

La Tabla 3 muestra las estadísticas descriptivas de las variables independientes utilizadas en este estudio. Se puede ver que el valor medio de endeudamiento es superior al 55\%, lo que significa que, en general, las empresas conciben la financiación externa como su fuente principal de recursos. Esto ha sido una característica común en las economías emergentes al atraer recursos, principalmente, de los prestamistas o acreedores financieros. No obstante, existen casos en que el nivel de endeudamiento supera el 100\% de sus activos (máximo=1.75). Esto es consecuencia de las pérdidas que tienen varias empresas para el año 2015, lo cual hace que su patrimonio sea negativo. Esto se refleja en el nivel medio de rentabilidad del -0.018.

Tabla 3

Descriptivos variables independientes

\begin{tabular}{lccccc}
\hline & $\mathrm{N}$ & Mínimo & Máximo & Media & Desviación estándar \\
\hline End & 31 & .2802 & 1.7502 & .550635 & .2915638 \\
Rent & 31 & -1.4102 & .1327 & -.018090 & .2634408 \\
CalAudit & 31 & 0 & 1 & .68 & .475 \\
Tam & 31 & 27238 & 122995950 & 7313932.61 & 22298299.020 \\
Inter & 31 & 0 & 1 & .45 & .506 \\
Edad & 31 & 1 & 34 & 22.84 & 12.496 \\
Var_NIIF & 31 & -.05 & .22 & .1010 & .07305 \\
\hline
\end{tabular}

Fuente: Elaboración propia. Datos obtenidos de SPSS 22 
Las compañías son en su mayoría auditadas por alguna de las Big Four y casi el 50\% de estas muestran algún nivel de internacionalización. Es de señalar que en promedio las empresas logran una antigüedad de dos décadas en el mercado de capitales colombiano. Sin embargo, se observa que existe una desviación significativa en esta variable (12.496), lo cual corresponde a la existencia de empresas que recientemente han sido listadas en la bolsa (mínimo=1) y otras que llevan más de treinta años participando activamente en el mercado de valores $($ máximo=34). Finalmente, el incremento porcentual en el grado de revelación entre los años 2014 y 2015 es en promedio para el conjunto de las empresas analizadas del 10\%.

La Tabla 4 muestra la matriz de correlación de Pearson entre todas las variables independientes y la dependiente utilizadas en el estudio. Una alta correlación entre las variables podría ser el indicio de un problema de multicolinealidad. Investigaciones previas (Alotaibi \& Hussainey, 2016; Liu \& Anbumozhi, 2009) indican que las variables tienen una alta correlación si esta es mayor que 0.80. Para las variables trabajadas los coeficientes de Pearson se encuentran por debajo de 0.80 , lo que indica que no existe un problema de colinealidad múltiple, lo que será verificado en la comprobación de los supuestos del modelo que se muestra más adelante. No obstante, existe un coeficiente que llama la atención y es el correspondiente a la relación rentabilidad y endeudamiento (-.727). Conceptualmente son variables vinculadas con las condiciones financieras de las empresas, pero cada una representa lógicas distintas, por lo que no se consideran cuestiones que puedan representar asuntos similares.

Tabla 4

Tabla de correlaciones de Pearson

\begin{tabular}{|c|c|c|c|c|c|c|c|c|c|}
\hline & & IR & End & Rent & CalAudit & Tam & Inter & Edad & Var_NIIF \\
\hline & & 015 & & & & & & & \\
\hline \multirow[t]{5}{*}{ IR2015 } & Correlación & 1 & .030 & -.040 & .070 & -.200 & $.545^{* *}$ & .268 & $.731^{* *}$ \\
\hline & de Pearson & & & & & & & & \\
\hline & Sig. (bilate- & & .872 & .831 & .710 & .280 & .002 & .144 & .000 \\
\hline & ral) & & & & & & & & \\
\hline & $\mathrm{N}$ & 31 & 31 & 31 & 31 & 31 & 31 & 31 & 31 \\
\hline \multirow[t]{5}{*}{ End } & Correlación & & 1 & $-.727^{* *}$ & -.233 & .124 & -.129 & -.227 & .127 \\
\hline & de Pearson & & & & & & & & \\
\hline & Sig. (bilate- & & & .000 & .206 & .508 & .489 & .220 & .495 \\
\hline & ral) & & & & & & & & \\
\hline & $\mathrm{N}$ & & 31 & 31 & 31 & 31 & 31 & 31 & 31 \\
\hline
\end{tabular}




\begin{tabular}{|c|c|c|c|c|c|c|c|}
\hline \multirow[t]{5}{*}{ Rent } & Correlación & 1 & .241 & -.079 & .104 & .251 & -.058 \\
\hline & de Pearson & & & & & & \\
\hline & Sig. (bilate- & & .192 & .674 & .577 & .174 & .756 \\
\hline & ral) & & & & & & \\
\hline & $\mathrm{N}$ & 31 & 31 & 31 & 31 & 31 & 31 \\
\hline \multirow[t]{5}{*}{ CalAudit } & Correlación & & 1 & .172 & .349 & -.267 & -.212 \\
\hline & de Pearson & & & & & & \\
\hline & Sig. (bilate- & & & .354 & .054 & .146 & .253 \\
\hline & & & & & & & \\
\hline & $\mathrm{N}$ & & 31 & 31 & 31 & 31 & 31 \\
\hline \multirow[t]{5}{*}{ Tam } & Correlación & & & 1 & .148 & -.214 & -.235 \\
\hline & de Pearson & & & & & & \\
\hline & Sig. (bilate- & & & & .426 & .249 & .203 \\
\hline & ral) & & & & & & \\
\hline & $\mathrm{N}$ & & & 31 & 31 & 31 & 31 \\
\hline \multirow[t]{5}{*}{ Inter } & Correlación & & & & 1 & .260 & .222 \\
\hline & de Pearson & & & & & & \\
\hline & Sig. (bilate- & & & & & .158 & .229 \\
\hline & & & & & & & \\
\hline & $\mathrm{N}$ & & & & 31 & 31 & 31 \\
\hline \multirow[t]{5}{*}{ Edad } & Correlación & & & & & 1 & .128 \\
\hline & de Pearson & & & & & & \\
\hline & Sig. (bilate- & & & & & & .494 \\
\hline & ral) & & & & & & \\
\hline & $\mathrm{N}$ & & & & & 31 & 31 \\
\hline \multirow[t]{5}{*}{ Var_NIIF } & Correlación & & & & & & 1 \\
\hline & de Pearson & & & & & & \\
\hline & Sig. (bilate- & & & & & & \\
\hline & ral) & & & & & & \\
\hline & $\mathrm{N}$ & & & & & & 31 \\
\hline
\end{tabular}

Fuente: Elaboración propia. Datos obtenidos de SPSS 22

Frente a la relación entre las variables independientes y la dependiente, se puede apreciar la existencia de una relación positiva y significativa entre la medida del $I R$ y la variable independiente internacionalización (.545) y el $I R$ y la Var_NIIF (.731); mientras que la relación con las demás variables (endeudamiento, rentabilidad, tamaño, calidad del auditor y edad) no resultan ser significativas a un nivel de $\mathrm{p}<0.05$. 


\section{Comprobación de supuestos del modelo de regresión}

Se han aplicado los test estadísticos para determinar la normalidad, la multicolinealidad, la independencia de los residuos y la heterocedasticidad, a partir del modelo planteado. De acuerdo con el test de Kolmogorov-Smirnov (K-S) se observa normalidad en la variable IR2015 al señalar que el p-valor (.200) no permite rechazar la hipótesis de normalidad, lo que se ratifica al aplicar el test de Shapiro-Wilk, el cual arroja un p-valor de .390 .

Para confirmar la ausencia de problemas de multicolinealidad entre las variables independientes, se realizó el cálculo del Factor de Inflación de la Varianza (VIF, por sus siglas en inglés): End=2.246; Rent=2.263; CalAudit=1.693; Tam=1.219; Inter=1.639; Edad=1.543; Var_NIIF=1.303, mostrando que cada uno de estos factores, se encuentra por debajo de 2.5 por lo que no se evidencia problemas de multicolinealidad entre las variables.

Asimismo, se aplicó la prueba de Durbin-Watson para efectos de detectar la independencia en los residuos (tabla 5). La prueba arroja un valor de 2.447, el cual al ubicarlo a partir del intervalo de 0.950 y 2.018 aplicable para una muestra de 31 observaciones, al nivel de significancia del $5 \%$ y un $\mathrm{K}=7$ ( $\mathrm{N}^{\circ}$ de regresores del modelo), permite determinar que el valor no se encuentra en las zonas de rechazo (entre 0 y dl=0.950; entre 4-dl y 4), por lo que las observaciones adyacentes no están correlacionadas.

Para realizar la prueba de heterocedasticidad al modelo, se realizó una prueba gráfica entre los residuos estandarizados y los valores predictivos estandarizados, visualizando una baja variabilidad de los residuos al cambio de los datos predictivos de los individuos. A su vez, se corrió un modelo de regresión teniendo como variable dependiente el valor de los residuos y las independientes propuestas en el modelo (forma de cálculo para cuando se utiliza SPSS), lo cual arrojó por medio de la prueba de ANOVA un p-valor de .777 el cual es mayor de $\mathrm{p}=0.05$, por lo que no existe significancia y se concluye que el modelo no tiene problemas de heterocedasticidad. En este orden de ideas, el modelo al cumplir con los supuestos evaluados resulta ser un buen predictor para el análisis planteado.

\section{Análisis del modelo de regresión}

De acuerdo con el análisis de regresión, el $\mathrm{R}^{2}$ ajustado es de .650 lo que significa que alrededor del $65 \%$ de las variaciones en el IR podrían explicarse a partir de las variables independientes trabajadas (Tabla 5). 
Tabla 5

Modelo de regresión

\begin{tabular}{|c|c|c|c|c|c|}
\hline Modelo & $\mathrm{R}$ & $\mathrm{R}$ cuadrado & $\begin{array}{l}\text { R cuadrado } \\
\text { ajustado }\end{array}$ & $\begin{array}{l}\text { Error estándar de } \\
\text { la estimación }\end{array}$ & Durbin-Watson \\
\hline 1 & $.855^{\mathrm{a}}$ & .731 & .650 & .0334565 & 2.447 \\
\hline
\end{tabular}

a. Predictores: (Constante), Var_NIIF, Rent, Inter, Tam, Edad, CalAudit, End

b. Variable dependiente: IR2015

Fuente: Datos obtenidos de SPSS 22

Asimismo, se encuentra que el modelo propuesto resulta ser explicativo, ya que de acuerdo con el análisis de ANOVA se muestra un p-valor de .000 (Tabla 6), lo que a un nivel de significancia de $\mathrm{p}<0.05$ permite deducir que es un buen modelo de predicción del $I R$.

Tabla 6

Prueba de Anova

\begin{tabular}{|c|c|c|c|c|c|c|}
\hline \multicolumn{2}{|c|}{ Modelo } & \multirow{2}{*}{$\begin{array}{l}\text { Suma de } \\
\text { cuadrados } \\
.070\end{array}$} & \multirow{2}{*}{$\begin{array}{l}\text { gl } \\
\quad 7\end{array}$} & \multirow{2}{*}{\begin{tabular}{c}
\multicolumn{1}{c}{$\begin{array}{c}\text { Media } \\
\text { cuadrática }\end{array}$} \\
.010
\end{tabular}} & \multirow{2}{*}{$\begin{array}{c}F \\
8.943\end{array}$} & \multirow{2}{*}{$\begin{array}{l}\text { Sig. } \\
.000^{\mathrm{b}}\end{array}$} \\
\hline 1 & Regresión & & & & & \\
\hline & Residuo & .026 & 23 & .001 & & \\
\hline & Total & .096 & 30 & & & \\
\hline
\end{tabular}

a. Variable dependiente: IR2015

b. Predictores: (Constante), Var_NIIF, Rent, Inter, Tam, Edad, CalAudit, End

Fuente: Datos obtenidos de SPSS 22

De acuerdo con la Tabla 7 que muestra los coeficientes del modelo, se hace evidente la relación significativa y positiva que tiene el $I R$ con el nivel de internacionalización de las empresas, por lo que se acepta $H 6$. Esto significa que una mayor internacionalización crea una presión por parte del mercado hacia una mayor probabilidad de que las empresas amplíen su información. Este hallazgo es coincidente con lo expuesto por Tran, Ngo, Phan, Do y Pham (2020), Skouloudis, Jones y Malesios (2014) y De Lima, De Lima, De Carvalho y Lima (2010), quienes han considerado que las empresas que operan en un escenario internacional tienden a revelar mayor cantidad de información de acuerdo con las reglas del sistema de negocios extranjeros. La necesidad de captar clientes internacionales, capital extranjero a un menor costo y mejorar su imagen pública (García \& Sánchez, 2006) puede ser una justificación razonable ante la relación observada entre la mayor información revelada y el nivel de internacionalización de las empresas. 


\begin{tabular}{llccccc}
\hline & & \multicolumn{5}{c}{ Coeficientes } \\
& & \multicolumn{7}{c}{ Coeficientes no estandarizados } & estandarizados & \\
\cline { 2 - 6 } & Modelo & $\mathrm{B}$ & Error estándar & Beta & $\mathrm{t}$ & Sig. \\
\hline \multirow{2}{*}{1 (Constante) } & .317 & .029 & & 10.968 & .000 \\
& End & -.005 & .031 & -.026 & -.158 & .876 \\
Rent & -.032 & .035 & -.147 & -.905 & .375 \\
CalAudit & .022 & .017 & .185 & 1.314 & .202 \\
Tam & $-2.572 \mathrm{E}-$ & .000 & -.101 & -.850 & .404 \\
& 10 & .015 & .321 & 2.323 & .029 \\
Inter & .036 & .001 & .161 & 1.199 & .243 \\
Edad & .001 & .095 & .649 & 5.261 & .000 \\
Var_NIIF & .502 & & & & \\
\hline
\end{tabular}

a. Variable dependiente: IR2015

Fuente: Elaboración propia. Datos obtenidos de SPSS 22

A su vez, se evidencia que, el mayor grado de revelación de la información para el año 2015 (IR2015), se explica de manera significativa y positivamente por el incremento porcentual generado en la revelación, a partir de la adopción de las NIIF (Var_NIIF), lo que coincide con los resultados de investigaciones previas (Hellman, Carenys \& Moya, 2018).

En este orden de ideas, las NIIF no solo generan un incremento en la revelación de información por la mayor exigencia de aspectos a divulgar, sino que se configura en un lineamiento que responde a las mejores prácticas internacionales y de aceptación en los mercados internacionales. Siendo evidente su uso como expresión, por parte de las empresas colombianas, de hacer uso de las mejores prácticas informativas para poder competir en el mundo de los negocios.

En cuanto a las demás variables explicativas se demuestra que existe una relación inversa entre el $I R$ con el endeudamiento, la rentabilidad, y el tamaño, pero sin que sean significativas, y positiva con la calidad del auditor y la edad, sin que se logre significancia al valor de $\mathrm{p}<0.05$. Por lo tanto, se rechazan $\mathrm{H} 2, \mathrm{H} 3, \mathrm{H} 4, \mathrm{H} 5$ y H7.

Estos últimos resultados no guardan relación con lo demostrado por estudios previos que han evaluado que el tamaño y el volumen de endeudamiento (De Lima, De Lima, De Carvalho \& Lima, 2010; Mir, Moreno \& Olmeda, 2006; Tawiah \& Boolaky, 2020), la rentabilidad (Zheng \& Chen, 2017) y el tipo de auditor (Tawiah \& Boolaky, 2020), son factores que determinan la mejora en la calidad de la información contable. 


\section{Conclusiones}

Colombia hace parte de los países emergentes que juegan un papel importante en la economía mundial y se ha convertido en un nicho de mercado potencial para diferentes grupos de inversores del resto del mundo, lo cual acarrea mejoras en las oportunidades de inversión. No obstante, el ser un país con mecanismos débiles de protección de los inversores, en comparación con los países desarrollados, hace que se requiera de la aplicación de prácticas corporativas internacionales que mejoren la confianza con sus grupos de interés.

La generación de información contable de alta calidad, a partir de la revelación de información, responde a esta necesidad, al propiciar condiciones de transparencia y comparabilidad que faciliten las relaciones comerciales y de inversión en el contexto internacional. En este sentido, las NIIF se plantean como lineamientos que favorecen la generación de información relevante para la toma de decisiones informadas por parte de los inversores, los prestamistas y de más grupos de interés. Para el IASB, la revelación de la información, por medio de las notas a los estados financieros, es fundamental para la toma de decisiones por parte de los usuarios externos, ya que ante las distintas alternativas de medición y reconocimiento de las partidas contables que traen las NIIF, se hace necesario ampliar la información que narra y explica la situación y desempeño financiero de las empresas.

En esta línea de pensamiento, el presente estudio evalúo el impacto que ha generado la aplicación de estos lineamientos en la revelación de información en empresas cotizadas en Colombia. Los resultados sugieren que ha existido una mejoría en la revelación de información una vez se han aplicado las NIIF. Investigaciones previas muestra que no existe un consenso sobre el impacto favorable de la adopción de las NIIF en la calidad de la información, ya que parece tener efectos diferenciados dependiendo el país o zona geográfica que se evalúe. Por lo tanto, el presente estudio aporta evidencia sobre los beneficios que ha traído las NIIF en la calidad de la información en el contexto de un país emergente como Colombia.

No obstante, se señala que aún existe un margen importante para que las empresas colombianas mejoren sus prácticas contables en materia de revelación de información, si se considera que los resultados obtenidos han estado por debajo de los promedios de cumplimiento de los requisitos de revelación obtenidos en los estudios previos. Por ende, hay expectativa que en la medida que avance el proceso de aplicación y los preparados de la información asimilen cada vez más los requerimientos de las NIIF se logre una mayor amplitud de la información revelada.

Es de señalar que la mejora en la revelación de la información no solo es consecuencia de la presión que genera la nueva regulación contable. Esta también se propicia por la presión de los mercados. Esto último se justificó y reflejó por medio de las características de las empresas, lo cual se relacionó con los resultados en la revelación de información. Se pudo 
evidenciar que el nivel de internacionalización que tienen las empresas influye positiva y significativamente en la amplitud de información que se dispone en las notas de los estados financieros. Esto indica que la presencia de las empresas en mercados foráneos, les obligada a divulgar información más completa de acuerdo con las reglas de información del sistema de negocios internacionales, por lo que tienen un incentivo de mercado que hace que la mejora en la revelación trascienda la mera obligación de aplicar las NIIF.

Contrario a esto, no se encontró relación entre los niveles de revelación de información a partir de las NIIF con variables como el tamaño, el nivel de endeudamiento, la rentabilidad, la calidad del auditor y la edad. Esto hace que no se obtenga evidencia empírica que refuerce lo que teóricamente se ha expuesto en la teoría de la agencia y bajo otros referentes teóricos.

Es de señalar que emisores en el ámbito mundial como el Instituto de Contadores Públicos de Escocia (ICAS) y el Instituto de Contadores Públicos de Nueva Zelanda (NZICA) han sugerido que las revelaciones requeridas en las NIIF se han vuelto demasiado gravosas y deberían reducirse (Saha, Morris \& Kang, 2019; Henderson, 2019), lo que puede estar incidiendo para que las empresas no cumplan plenamente con los requisitos de divulgación y crean que el divulgar no agrega valor. Por lo tanto, resultados como los evidenciados sobre la cantidad y los detalles que se proporcionan en las notas, podrían dar elementos adicionales a los emisores de las normas, como el IASB, para efectos de contemplar los ajustes en su proyecto sobre los principios de divulgación de las NIIF.

Asimismo, los resultados de este estudio al mostrar qué requisitos de divulgación se cumplen y cómo esto se asocia con los incentivos de divulgación de las empresas, resulta ser de relevancia para los supervisores, ya que sirven de base para posibilitar un seguimiento frente al cumplimiento de la norma. A su vez, el estudio realizado resulta de interés para las empresas en la medida que, al ser una observación externa, muestra cómo se ha avanzado en el proceso de implementación de las NIIF y las recomendaciones que se pueden tener al respecto.

El estudio contribuye en la ampliación de la investigación contable, ya que la mayoría de los estudios se han enfocado en evaluar el efecto de las NIIF en la calidad de la información contable a partir del estudio de la manipulación del resultado, el reconocimiento oportuno de pérdidas y la relevancia valorativa (Christensen, Lee, Walker \& Zeng, 2015; Cardona, Gómez \& Cano, 2019; Eng, Lin \& Neiva, 2019), dejando en un segundo plano la relevancia informativa, es decir, en analizar las consecuencias en la revelación de la información. Este tema no deja de ser menor en el estudio de la regulación contable, ya que puede entenderse como un tema que pasa por las preocupaciones políticas al interior de las organizaciones para decidir qué revelar, lo cual es quizás distinto a "los problemas de medición" que en gran medida son un tema técnico y no político (Coy, Fischer, \& Gordon, 2001).

Finalmente, se hace necesario el desarrollo de estudios posteriores que puedan ampliar el análisis en otras partidas contables y en distintos tipos de empresas, como las pymes y las 
pertenecientes al tercer sector de la economía. A su vez, se pueden posibilitar estudios que hagan seguimiento en el tiempo al cumplimiento de los requerimientos de revelación, más aún, cuando se está en proceso de mejorar los requerimientos de cada uno de los estándares y de la definición global de principios de revelación por parte del IASB.

\section{Referencias}

Abdul, Z. \& Haniffa, R. (2020). The impact of accounting reform on accounting quality: evidence from Nigeria. Journal of International Financial Management \& Accounting, 31 (2), 169-190. https://doi.org/10.1111/ jifm.12112

Ahmed, J. \& Karim, W. (2005). Compliance to international accounting standards in Bangladesh: a survey of annual reports. The Bangladesh Accounting, 40 (1), 23-24.

Aksu, M. \& Kosedag, A. (2006). Transparency and disclosure scores and their determinants in the Istanbul stock exchange. Corporate Governance An International Review, 14 (4), 277-296. https://doi.org/10.1111/j.14678683.2006.00507.x

Albu, N., Albu, C., \& Gray, S. (2020): Institutional factors and the impact of international financial reporting standards: the Central and Eastern European experience, Accounting Forum, 44(3): 184-214. https://doi.org/10.10 80/01559982.2019.1701793

Alonso, M. (2009). La transparencia de las empresas en internet para la confianza de los accionistas e inversores: un análisis empírico. Cuadernos de Administración, 22 (38), 11-30. Disponible en: https://revistas.javeriana. edu.co/index.php/cuadernos_admon/article/view/3860 Consultado: 02/02/2018

Alotaibi, K. \& Hussainey, K. (2016). Determinants of CSR disclosure quantity and quality: evidence from non-financial listed firms in Saudi Arabia. International Journal of Disclosure and Governance, 13 (4), 364-393. https://doi.org/10.1057/jdg.2016.2

Angla, J. (2013). La investigación empírica sobre la divulgación de la información financiera: revisión de la literatura. Revista Internacional Legis de Contabilidad y Auditoría, 14, 11-61.

Agyei, B. (2017). The relationship between corporate governance, corruption and forward-looking information disclosure: a comparative study. Corporate Governance: The International Journal of Business in Society, 17 (2): 284-304. https://doi.org/10.1108/CG-11-2015-0150

Armitage, S. \& Marston, C. (2008). Corporate disclosure, cost of capital and reputation: evidence from finance directors. The British Accounting Review, 40 (4): 314-336. https://doi.org/10.1016/j.bar.2008.06.003

Barth, M., Landsman, W., \& Lang, M. (2008). International Accounting Standards and accounting quality. Journal of Accounting Research, 46 (3), 467-498. https://doi.org/10.1111/j.1475-679X.2008.00287.x

Bauman, M. (2013). The adequacy of fixed asset disclosures under U.S. GAAP. Research in Accounting Regulation, 25 (2), 149-156. https://doi.org/10.1016/j.racreg.2013.08.002

Bonsón, E. \& Escobar, T. (2004). La difusión voluntaria de información financiera en internet. Un análisis comparativo entre Estados Unidos, Europa del Este y la Unión Europea. Spanish Journal of Finance and Accounting, 33 (123): 1063-1101. https://doi.org/10.1080/02102412.2004.10779539

Braam, G., Uit, L., Hauck, M., \& Huijbregts, M. (2016). Determinants of corporate environmental reporting: the importance of environmental performance and assurance. Journal of Cleaner Production, 129, 724-734. https:// doi.org/10.1016/j.jclepro.2016.03.039

Bravo, F. (2016). Forward-looking disclosure and corporate reputation as mechanisms to reduce stock return volatility. Revista de Contabilidad, 19 (1), 122-131. https://doi.org/10.1016/j.rcsar.2015.03.001

Bravo, F., Abad, M., \& Trombetta, M. (2009). Disclosure indices design: does it make a difference? Spanish Accounting Review, 12 (2), 253-278. https://doi.org/10.1016/S1138-4891(09)70008-1 
Brochet, F., Jagolinzer, A., \& Riedl, E. (2013). Mandatory IFRS adoption and financial statement comparability. Contemporary Accounting Research, 30 (4): 1373-1400. https://doi.org/10.1111/1911-3846.12002

Cardona, J., Gómez, A., \& Cano, A. (2019). Impacto de los estándares internacionales de información financiera en la calidad contable: evidencia desde Latinoamérica y el Caribe. Contaduría y Administración, 64 (4), 1-37. http://dx.doi.org/10.22201/fca.24488410e.2018.1669

Carreño, J. (2015). La importancia de las revelaciones en los estados financieros bajo NIIF para la toma de decisiones, Trabajo de grado de Especialización, Facultad de Ciencias Económicas, Universidad Militar Nueva Granada. Disponible en: http://hdl.handle.net/10654/7236 Consultado: 02/02/2018

Católico, D., Cely, V. \& Pulido, J. (2013). Revelación de la información financiera sobre propiedad, planta y equipo en empresas industriales cotizadas en Colombia, Cuadernos de Contabilidad, 14 (36), 943-970. Disponible en: https://revistas.javeriana.edu.co/index.php/cuacont/article/view/7909 Consultado: 11/11/2017

Christensen, H., Lee, E., Walker, M., \& Zeng, Ch. (2015). Incentives or Standards: what determines accounting quality changes around IFRS adoption? European Accounting Review, 24 (1), 31-61. https://doi.org/10.1080/ 09638180.2015 .1009144

¿Cómo está compuesta la economía colombiana? (2015, Septiembre 29). Disponible en: https://www.dinero.com/ economia/articulo/composicion-economia-colombiana-2015/214054. Consultado: 15/09/2018

Coluccia, D., D’Amico, E., Fontana, S., \& Solimene, S. (2017). A cross-cultural perspective of voluntary disclosure: Italian listed firms in the stakeholder global context. European J. International Management, 11 (4), 430-451. https://doi.org/10.1504/EJIM.2017.085582

Cooke, T. (1989). Voluntary corporate disclosure by Swedish Companies. Journal of International Financial Management and Accounting, 1 (2), 171-195. https://doi.org/10.1111/j.1467-646X.1989.tb00009.x

Consejo Técnico de la Contaduría Pública (2009). Presentación de estados financieros con base en estándares internacionales de contabilidad e información financiera IAS-IFRS. Revista Internacional Legis de Contabilidad \& Auditoría, 38, 67-132

Coy, D., Fischer, M., \& Gordon, T. (2001). Public accountability: a new paradigm for college and university annual reports. Critical Perspectives on Accounting, 12 (1), 1-31. https://doi.org/10.1006/cpac.2000.0416

Cutillas, M., Sánchez, J., \& Yagüe, J. (2016). The effects of IFRS on net income and earnings components: value relevance, persistence, and predictive value. Spanish Journal of Finance and Accounting, 45 (3), $365-388$. http://dx.doi.org/10.1080/02102412.2016.1198562

Dayanandan, A., Donker, H. Ivanof, M., \& Karahan, G. (2016). IFRS and accounting quality: legal origin, regional, and disclosure impacts. International Journal of Accounting and Information Management, 24 (3), $296-316$. https://doi.org/10.1108/IJAIM-11-2015-0075

De Lima, V., De Lima, G., De Carvalho, N. \& Lima, I. (2010). Toward IFRS: economic consequences of accounting convergence in an emerging economy. Research in Accounting in Emerging Economies, 10, 251 - 295. https:// doi.org/10.1108/S1479-3563(2010)0000010015

De Moura, A., Altuwaijri, A., \& Gupta, J. (2020). Did mandatory IFRS adoption affect the cost of capital in Latin American countries? Journal of International Accounting, Auditing and Taxation, 38, 100301. https://doi-org/10.1016/j.intaccaudtax.2020.100301

Departamento Administrativo Nacional de Estadística (DANE) (2017). Cuentas Trimestrales - Colombia, Producto Interno Bruto (PIB), Cuarto Trimestre de 2016. Disponible en: http://www.dane.gov.co/files/investigaciones/ boletines/pib/cp_PIB_IVtrim16_oferta.pdf Consultado: 15/07/2018

El-Helaly, M., Ntim, C., \& Al-Gazzar, M. (2020). Diffusion theory, national corruption and IFRS adoption around the world. Journal of International Accounting, Auditing and Taxation, 38, 100305. https://doi.org/10.1016/j. intaccaudtax.2020.100305

Eng, L, Lin, J., \& Neiva, J. (2019). International Financial Reporting Standards adoption and information quality: evidence from Brazil. Journal of International Financial Management \& Accounting, 30 (1), 5-29. https://doi. org/10.1111/jifm.12092 
Firmino, C., Nogueira, K., Cavalcante, D., Magalhães, S., \& Rodrigues, V. (2019). Accounting information quality of Latin American Firms: The Influence of the Regulatory Environment. Revista Evidenciação Contábil \& Finanças, 7 (2), 40-60. https://doi.org/10.22478/ufpb.2318-1001.2019v7n2.41086

Frías, J., Rodríguez. L., \& García, I. (2014). Explanatory factors of integrated sustainability and financial reporting. Business Strategy and the Environment, 23, 56-72. https://doi.org/10.1002/bse.1765

García, E. \& Sánchez, J. (2006). Un estudio meta-analítico de los factores determinantes de la revelación de información. Spanish Journal of Finance and Accounting, 35 (132), 761-788. https://doi.org/10.1080/02102412.2 006.10779605

Giner, B. (1995). La divulgación de la información financiera: una investigación empírica. España: Instituto de Contabilidad y Auditoría de Cuentas, ICAC.

Giner, B. (1997). The influence of company characteristics and accounting regulation on information disclosed by spanish firms. The European Accounting Review, 6 (1), 45-68. https://doi.org/10.1080/096381897336863

Grajales, J., Cuevas, J., \& Usme, W. (2015). Balance de las publicaciones sobre IFRS en dos revistas colombianas, Revista Internacional Legis de Contabilidad \& Auditoría, 64, 83-120.

Griffin, P., Lont, D., \& Sun, Y. (2009). Governance regulatory changes, International Financial Reporting Standards adoption, and New Zealand audit and non-audit fees: empirical evidence. Accounting \& Finance, 49, 697-724. https://doi.org/10.1111/j.1467-629X.2009.00310.x

Grosman, A., Smith 1., \& Tervo, W. (2013). Measuring the impact of international reporting standards on market performance of publicly traded companies. Advances in Accounting, 29 (2), 343-349. https://doi.org/10.1016/j. adiac.2013.09.001

Harakeh, M., Lee, E., \& Walker, M. (2019). The differential impact of IFRS adoption on aspects of seasoned equity offerings in the UK and France. Accounting in Europe, 16 (1), 106-138. https://doi.org/10.1080/17449480.20 18.1531141

Hellman, N., Carenys, J., \& Moya, S. (2018). Introducing more IFRS principles of disclosure - will the poor disclosers improve? Accounting in Europe, 15 (2), 242-321. https://doi.org/10.1080/17449480.2018.1476772

Henderson, E. (2019). Users' perceptions of usefulness and relevance of financial statement note disclosures and information overload. International Journal of Business, Accounting, \& Finance, 13 (1), 41-56. Disponible en: https://international.vlex.com/vid/users-perceptions-of-usefulness-782632133 Consultado: 02/07/2019

Holthausen, R. \& Watts, R. (2001). The relevance of the value-relevance literature for financial accounting standard setting. Journal of Accounting and Economics, 31 (1-3), 3-75. https://doi.org/10.1016/S0165-4101(01)000295

Hoque, Z. (2006). Methodological Issues in Accounting Research: Theories and Methods. London: Spiramus.

Hutagaol, M., Valentincic, A., \& Warganegara, D. (2019). Earnings quality and market values of Indonesian listed firms. Australian Accounting Review, 29 (1), 95-111. https://doi.org/10.1111/auar.12234

Iatridis, G. (2008). Accounting disclosure and firms' financial attributes: evidence from the UK stock market. International Review of Financial Analysis, 17 (2), 219-241. https://doi.org/10.1016/j.irfa.2006.05.003

Iatridis, G. (2010). International Financial Reporting Standards and the quality of financial statement information. International Review of Financial Analysis, 19, 193-204. https://doi.org/10.1016/j.irfa.2010.02.004

International Accounting Standards Board (2010). El marco conceptual para la información financiera. London: International Accounting Standards Committee Foundation.

International Accounting Standards Board (2017a). Iniciativa de Información a Revelar - Principios de Información a Revelar. London: International Accounting Standards Committee Foundation.

International Accounting Standards Board (2017b). Realización de Juicios sobre Materialidad o Importancia Relativa. Documento de Práctica $\mathrm{N}^{\circ}$ 2. London: International Accounting Standards Committee Foundation.

Ioana, A., Tiron, A., \& Pali, S. (2014). Compliance of non-current assets with IFRS requirements concerning the information disclosure - case study. Procedia Economics and Finance, 15, 1391-1395. https://doi.org/10.1016/ S2212-5671(14)00603-0 
Key, K., \& Kim, J. (2020). IFRS and accounting quality: additional evidence from Korea. Journal of International Accounting, Auditing and Taxation. 39, 100306. https://doi-org/10.1016/j.intaccaudtax.2020.100306

Larrán, J. \& García, E. (2004). Costes, beneficios y factores ligados a la política de divulgación de información financiera. Revista de Contabilidad, 7 (14), 75-111. Disponible en: https://revistas.um.es/rcsar/article/ view/387391 Consultado: 15/08/2018

Lasso, G., Vargas, C. \& Ruano, C. (2018). Efecto patrimonial del proceso de convergencia contable en las Pymes colombianas. Estudios Gerenciales, 34 (146), 99-113. https://doi.org/10.18046/j.estger.2018.146.2814

Liu, X. \& Anbumozhi, V. (2009). Determinant factors of corporate environmental information disclosure: an empirical study of Chinese listed companies. Journal of Cleaner Production, 17, 593-600. https://doi.org/10.1016/j. jclepro.2008.10.001

Mantilla, S. (1996). Normas técnicas sobre revelaciones. En J. Jiménez, C. Maldonado, E. Espinosa, L. Ramírez, S. Mantilla, J. Cardona, R. Vásquez, H. Madrid, J. Carvhalo y F. Torres (Ed.), Perspectivas y aplicación de la contabilidad en Colombia (pp. 587-718). Colombia: Departamento de Publicaciones Cámara de Comercio de Bogotá.

Mantilla, S. (2002). Guía para la inserción contable colombiana en los escenarios internacionales. Bogotá D.C.: Pontifica Universidad Javeriana

Mi, J., Sheng, H., \& Elrod, C. (2016). Financing, reputation and information disclosure quality in the Chinese market. Applied Economics, 48 (9), 789-798. http://dx.doi.org/10.1080/00036846.2015.1088140

Mnif, Y. \& Znazen, O. (2020). Corporate governance and compliance with IFRS 7: the case of financial institutions listed in Canada. Managerial Auditing Journal, 35 (3), 448-474. https://doi.org/10.1108/MAJ-08-2018-1969

Mir, C., Moreno, D., \& Olmeda, I. (2006). Determinantes de la revelación de información sobre derivados financieros en el mercado español, Ponencia, V Workshop de Investigación Empírica en Contabilidad Financiera, REFC.

Mongrut, S., \& Winkelried, D. (2019). Unintended effects of IFRS adoption on earnings management: the case of Latin America. Emerging Markets Review, 38, 377-388. https://doi-org/10.1016/j.ememar.2018.11.004

Nassreddine, G. (2016). Determinants of financial information disclosure: a visualization test by cognitive mapping technique. Journal of Economics and Finance, 21, 8-13. http://dx.doi.org/1 0.1016/j.jefas.2016.03.002

Pascan, I. (2015). Measuring the effects of IFRS adoption on accounting quality: a review. Procedia Economics and Finance, 32, 580-587. https://doi.org/10.1016/S2212-5671(15)01435-5

Rico, C., Montoya, L., Franco, B., \& Laverde, M. (2020). La comparabilidad de la información financiera en Colombia tras su convergencia con los IFRS. El caso de las propiedades, planta y equipo de las empresas cotizantes. Innovar: Revista de Ciencias Administrativas y Sociales, 30 (76), 91-104. https://doi-org/10.15446/ innovar.v30n76.85215

Saha, A., Morris, R., \& Kang, H. (2019). Disclosure overload? An empirical analysis of international financial reporting standards disclosure requirements. Abacus, 55 (1), 205-236. https://doi.org/10.1111/abac.12148

Schnackenberg, A. \& Tomlinson, E. (2016). Organizational transparency: a new perspective on managing trust in organization-stakeholder relationships. Journal of Management, 42 (7), 1784-1810. https://doi. org/10.1177/0149206314525202

Skouloudis, A., Jones, N., \& Malesios, Ch. (2014). Trends and determinants of corporate non-financial disclosure in Greece. Journal of Cleaner Production, 68, 174-188. http://dx.doi.org/10.1016/j.jclepro.2013.12.048

Soderstrom, N. \& Sun, K. (2007). IFRS adoption and accounting quality: a review. European Accounting Review, 16 (4): 675-702. https://doi.org/10.1080/09638180701706732

Subramaniam. N. (2006). Agency theory and accounting research: an overview of some conceptual and empirical issues. En Hoque, Z. (Ed.), Methodological Issues in Accounting Research: Theories and Methods. (pp. 55-82). London: Spiramus.

Superintendencia de Sociedades (2014). Informe ESFA Empresas del Grupo 1 en el sector real. Disponible en: https://www.supersociedades.gov.co/delegatura_aec/regulacion_contable/construccion_conjunta/INFORME-ESFA-2014-Grupo1.pdf Consultado: 15/03/2018 
Superintendencia de Sociedades (2015). Análisis de impacto. Empresas grupo 1 a 31 de diciembre de 2014. Disponible en: https://www.supersociedades.gov.co/delegatura_aec/regulacion_contable/memorias_encuentro_niif/PRESENTACION\%20ANALISIS\%20DE\%20IMPACTO\%20NIIF\%2017\%20SEPT\%202015.pdf Consultado: 15/03/2018

Tawiah, V. \& Boolaky, P. (2020). Consequences and determinants of IFRS convergence in India. International Journal of Accounting \& Information Management, 28 (2), 303-322. https://doi.org/10.1108/IJAIM-06-2019-0062

Tawiah, V. \& Boolaky, P. (2019). Determinants of IFRS compliance in Africa: analysis of stakeholder atributes. International Journal of Accounting \& Information Management, 27 (4), 573-599. https://doi.org/10.1108/ IJAIM-09-2018-0110

Tran, M., Ngo, T., Phan, T., Do, D., \& Pham, T. (2020). Determinants influencing the conversion of financial statements: the case of multinational firms in Vietnam. The Journal of Asian Finance, Economics and Business, 7 (3), 17-18. https://doi.org/10.13106/jafeb.2020.vol7.no3.17

Wijayana, S., \& Gray, S. (2019). Institutional factors and earnings management in the Asia-Pacific: is IFRS adoption making a difference? Management International Review (MIR), 59 (2), 307-334. https://doi-org/10.1007/ s11575-018-0371-1

Zeng, S., Xu, X., Yin, H., \& Tam, C. (2012). Factors that drive chinese listed companies in voluntary disclosure of environmental information. Journal of Business Ethics, 109 (3), 309-321. https://doi.org/10.1007/s10551011-1129-x

Zheng, X. \& Chen, J. (2017). Financial reporting quality in China: a perspective of qualitative characteristics. Transformations in Business \& Economics, 16 (3), 148-163. 